\title{
IV. Ausgabenpolitik
}

\section{Das Wagnersche Gesetz}

Adolph Wagner, der Nestor der deutschen Finanzwissenschaft, hat bereits vor fast 150 Jahren sein berühmtes „Gesetz der wachsenden Ausdehnung der Staatsthätigkeit" formuliert. Weitsichtig erkannte er, dass die öffentlichen Aufgaben - und damit auch die Ausgaben - im Zuge der von ihm begrüßten Entwicklung vom Ordnungs- zum Wohlfahrtsstaat zunehmen würden. Es lohnt sich, ihn selbst zu Wort kommen zu lassen:

„Wo wir hinsehen, treten überall [...] neue Aufgaben [auf] oder müssen die alten auf neue Weise, besser und vollkommener ausgeführt werden. [...] Wir bedürfen einer gesicherten Rechtsordnung im Innern und nach Aussen. [...] Wir bedürfen nicht minder zahlloser fördernder öffentlicher Thätigkeiten im Interesse der Volkswirthschaft und Cultur. Die Privatthätigkeit reicht auch hier immer weniger aus. [...] Ich erinnere nur an drei grosse Gebiete, die gegenwärtig in unseren Culturstaaten überall im Vordergrund stehen: an das Unterrichtswesen mit seinen immer grösseren und feineren Bedürfnissen, an das Verkehrswesen, die Wege und Verkehrsanstalten, Post, Telegraphen, Eisenbahnen usw., und an speciell städtische Bedürfnisse, der Reinlichkeit, Gesundheitspflege, Bequemlichkeit, der Versorgung mit Lebensmitteln usw., daher Anstalten der Wasserleitung, Canalisirung, Gasbeleuchtung - vielleicht bald electrische Beleuchtung -, öffentliche Gärten, Markthallen, Viehhöfe usw. Ueberall hat hier bereits und wird immer mehr die öffentliche Thätigkeit des Staats, Kreises, der Gemeinde Platz greifen - was nichts anderes heisst, als Steigerung der Gemeinwirthschaft, mithin des Finanzbedarfs [...]. ${ }^{\text {"1 }}$

$\mathrm{Zu}$ Recht ist gegen das Wagnersche Gesetz eingewandt worden, dass die öffentlichen Ausgaben keineswegs zwangsläufig steigen müssen. Das zeigt der Verlauf der deutschen Staatsquote, also dem Anteil der öffentlichen Ausgaben an der Wirtschaftsleistung, seit 1900 deutlich. Zwar sind die Staatsausgaben insgesamt stark gewachsen, doch gab es immer wieder auch Phasen einer gegenläufigen Entwicklung, wie das folgende Schaubild zeigt. Das Jahr 1938 fällt dabei etwas aus dem Rahmen, da die Vorbereitung des Angriffskriegs die Staatsquote bereits Richtung 50 Prozent gedrückt hat, während der Anteil der Sozialausgaben stark sank. Ansonsten hat die Sozialleistungsquote die Entwicklung der Staatsquote maßgeblich beeinflusst. Der generelle Trend des Anstiegs der Sozialleistungen setzte sich nach dem Zweiten Weltkrieg fort. Zunächst mussten die sozialen Folgekosten des Kriegs finanziert

${ }^{1}$ Adolph Wagner, Ueber die schwebenden deutschen Finanzfragen, in: Zeitschrift für die gesamte Staatswissenschaft 35 (1879), S. 68-114, hier S.77f. 
werden. Dann folgte bereits in den 1950er Jahren der Ausbau des Sozialstaats. Im Unterschied zur Weimarer Republik, die sich ebenfalls auf dem Weg zum Wohlfahrtsstaat befand, konnte sich die Bonner Republik das auch leisten, zumindest in den ersten 25 Jahren ihres Bestehens. Das „Wirtschaftwunder" machte es möglich. Aufgrund der hohen Wachstumsraten und der mit Ausnahme der Sozialpolitik eher sparsamen Haushaltsführung sank die Staatsquote in den 1950er Jahren sogar leicht. In den folgenden zwei Jahrzehnten kehrte sich der Trend dafür um. Die Jahre zwischen 1969 und 1975 wurden „zur Phase der größten Beschleunigung sozialstaatlicher Expansion“2.

Abbildung 5: Entwicklung der Staats- und Sozialleistungsquote, 1900-2010³

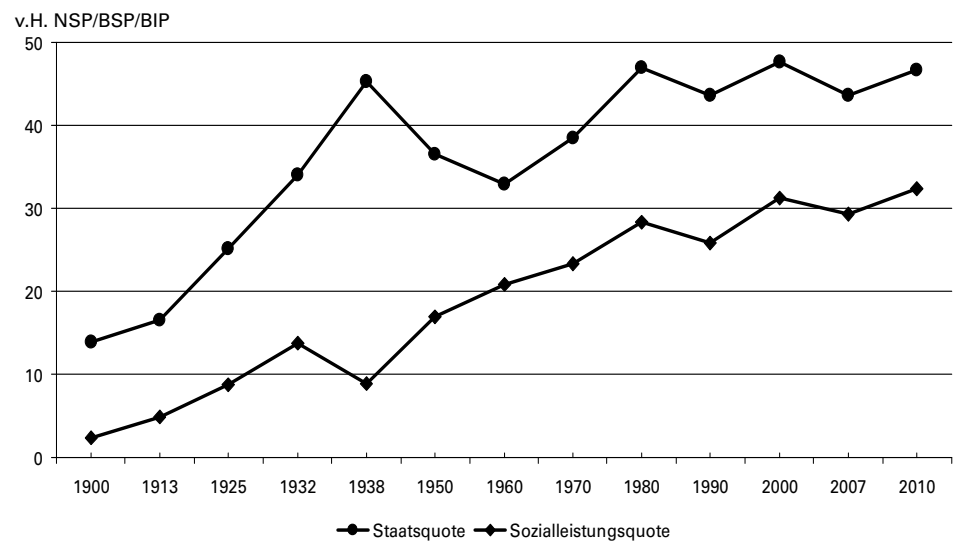

${ }^{2}$ Anselm Doering-Manteuffel/Lutz Raphael, Nach dem Boom. Perspektiven auf die Zeitgeschichte seit 1970, Göttingen ${ }^{2} 2010$, S. 47.

${ }^{3}$ Staatsquoten (Nettosozialprodukt zu Faktorpreisen) für die Jahre von 1900 bis 1950: vgl. Horst Claus Recktenwald, Umfang und Struktur der öffentlichen Ausgaben in säkularer Entwicklung, in: Handbuch der Finanzwissenschaft, hrsg. von Fritz Neumark unter Mitwirkung von Norbert Andel und Heinz Haller, Bd. 1, Tübingen ${ }^{3}$ 1977, S. 713 752, hier S.719; Staatsquoten für die Jahre 1960 bis 2010: vgl. Monatsbericht des Bundesministeriums der Finanzen vom Januar 2011, S. $81 \mathrm{f}$; S Sozialleistungsquote für das Jahr 1913: vgl. Johannes Frerich/Martin Frey, Handbuch der Geschichte der Sozialpolitik in Deutschland, Bd. 1: Von der vorindustriellen Zeit bis zum Ende des Dritten Reiches, München/Wien 1993, S.175; für die restlichen Jahre bis 1950: vgl. Heinz Lamper/Jörg Althammer, Lehrbuch der Sozialpolitik, Berlin ${ }^{8} 2007$, S.510 (Tabelle 18.1.); für die Jahre ab 1960: Sozialbericht 2009, hrsg. vom Bundesministerium für Arbeit und Soziales, Bonn 2009, Tabelle I-1 und S. 256 (Jahr 2010). 
Maßgeblich verursacht durch den Ausbau der Sozialleistungen erreichte die Staatsquote Anfang der 1980er Jahre fast den magischen Wert von 50 Prozent. Das hatte - ähnlich wie in den Jahren der Weltwirtschaftskrise - mit der Rezession zu tun, da die Sozialausgaben infolge der Massenarbeitslosigkeit stiegen, während das Bruttoinlandsprodukt fiel. Daher steigt die Staatsquote meist in konjunkturell schwierigen Zeiten, selbst wenn eine harte Sparpolitik wie zwischen 1930 und 1932, betrieben wird. Umgekehrt sinkt die Staatsquote meist in wirtschaftlich guten Zeiten. Das war von 1984 bis 1989 so, und das ist auch zwischen 2005 und 2008 so gewesen. Beide Phasen waren von einem robusten Wirtschaftswachstum, rückläufiger Arbeitslosigkeit und einem moderaten Sozialabbau geprägt. In logischer Konsequenz sank die Staatsquote 2007 auf unter 44 Prozent ${ }^{4}$. Das war einer der niedrigsten Werte seit fast vier Jahrzehnten; auch im internationalen Vergleich lag die Bundesrepublik damit im unteren Bereich. So weist Frankreich nicht zuletzt infolge der etatistischen Tradition seit langem eine Staatsquote von über 50 Prozent auf. Spitzenreiter sind aufgrund des gut ausgebauten sozialen Sicherungssystems seit eh und je die skandinavischen Länder. Großbritanniens Staatsquote lag 2007 das erste Mal seit langer Zeit über der deutschen. Die USA hatten im gleichen Jahr mit 37 Prozent eine deutlich niedrigere Staatsquote. Dabei ist jedoch zu berücksichtigen, dass ein nicht geringer Teil der Sozialausgaben von den Amerikanern privat finanziert werden muss. Insofern ist die Staatsquote der USA sogar bemerkenswert hoch, was vor allem an den Militärausgaben liegt. Eine deutsche Besonderheit ist die hohe Sozialleistungsquote, die international nur von Dänemark, Schweden und Frankreich übertroffen wird. Das verdeutlicht, wie stark Deutschland seinen Sozialstaat ausgebaut hat.

\section{Die Entwicklung der Ausgabenstruktur}

Die Entwicklung Deutschlands vom Militär- zum Wohlfahrtsstaat wird in Abbildung 6 sehr deutlich. Während der Anteil der Militärausgaben am nationalen Haushalt von 79 Prozent auf zehn Prozent sank, vervielfachten sich die Ausgaben für Soziales, Arbeitsmarkt und Renten von drei auf 52 Prozent. Aus Abbildung 6 wird deutlich, dass der Bundeshaushalt spätestens seit 2007 nicht nur ein Schuldenproblem, sondern auch ein Qualitätsproblem hat. Die Zinsausgaben und der Zuschuss an die Rentenversicherung machten

\footnotetext{
${ }^{4}$ Vgl. Monatsbericht des Bundesministeriums der Finanzen vom Januar 2011, S. 81; zum Folgenden vgl. ebenda, S. 90.
} 
Abbildung 6: Ausgabenstruktur des Reichs-/Bundeshaushalts in den Jahren 1913, 1963, 1983 und $2007^{5}$
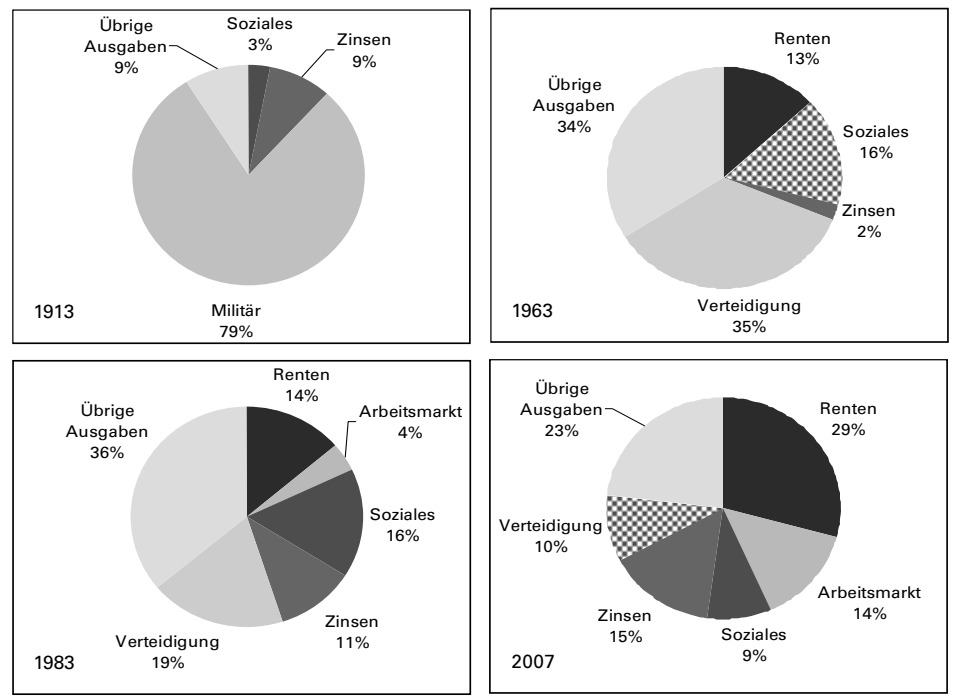

allein 44 Prozent des Haushalts aus. In logischer Konsequenz halbierte sich der Anteil der diskretionären, also politisch beeinflussbaren Ausgaben im Bundeshaushalt von knapp 40 Prozent 1970 auf 19,5 Prozent 2007 ${ }^{6}$. Der Anteil der Zins- und Rentenausgaben wird in Zukunft aufgrund der demographischen Entwicklung sowie der Schulden- und Zinsentwicklung deutlich steigen. Zins- und Rentenausgaben finanzieren „Rentner [...] und Rentiers ${ }^{\text {“7 }}$, sind also alles andere als innovations- und zukunftsfördernd. Sie alimentieren ausschließlich die Vergangenheit in Form der früheren Kreditaufnahme und der vormals erworbenen Rentenansprüche. Anders ausgedrückt: Die Qualität der Staatsausgaben ist schlecht.

${ }^{5}$ Für das Jahr 1913 vgl. Ullmann, Steuerstaat, S. 62; für die Jahre 1963, 1983 und 2007 vgl. die Bundeshaushalte der entsprechenden Jahre.

${ }^{6}$ Wolfgang Streeck/Daniel Mertens, Politik im Defizit: Austerität als fiskalpolitisches Regime, in: Der moderne Staat 3 (2010), S. 7-29, hier S. 14 (Tabelle 1).

${ }^{7}$ Hans Günter Hockerts, Der deutsche Sozialstaat. Entfaltung und Gefährdung seit 1945, Göttingen 2011, S. 17. 


\section{Sozialausgaben}

Bis 1957 sicherten die Altersrenten nur einen bescheidenen Lebensunterhalt und besaßen „den Charakter einer ärmlichen Überlebenshilfe ${ }^{\text {c8 }}$. Wie in der gesamten Menschheitsgeschichte zuvor hatten vor allem die Kinder für ihre alten Eltern zu sorgen. Kinderlose waren in der Regel auf kommunale oder kirchliche Fürsorge angewiesen. Die Einführung der dynamischen Rente im Jahr 1957 änderte dies grundlegend. Seitdem geht der Renteneintritt nicht mehr zwangsläufig mit einem deutlich niedrigeren Lebensstandard einher; die Rente ist im Gegenteil zumindest bis zur Rentenreform von 2004 statussichernder Lohnersatz gewesen ${ }^{9} .1957$ wurden die Renten um 72 Prozent für Angestellte und 65 Prozent für Arbeiter erhöht ${ }^{10}$, was einem sozialpolitischen Quantensprung gleichkam und den Anfang vom Ende der Altersarmut als Massenphänomen bedeutete. Um dieses Niveau dauerhaft zu gewährleisten, koppelte der Gesetzgeber die Entwicklung der Renten an die Bruttolohnsteigerungen. Die Finanzierung der Rentenversicherung erfolgt seitdem durch ein Umlagesystem, das den großen Vorteil hat, dass die von den Arbeitnehmern und Arbeitgebern zu zahlenden Beiträge sofort in die Rentenkasse fließen ${ }^{11}$. Über die richtige Finanzierung der Rentenversicherung herrschten im Laufe der Jahrzehnte unterschiedliche Ansichten $^{12}$. Vor dem Krieg bestand über die Notwendigkeit einer Kapitaldeckung Konsens. Hingegen favorisierte 1957 die Mehrzahl der Experten und

${ }^{8}$ Ebenda, S.71. Vgl. Winfried Schmähl, Die Einführung der „dynamischen Rente“ 1957. Gründe, Ziele und Maßnahmen - Versuch einer Bilanz, Bremen 2007, S. 9.

${ }^{9}$ Vgl. Hans Günter Hockerts, Abschied von der dynamischen Rente. Über den Einzug der Demografie und der Finanzindustrie in die Politik der Alterssicherung, in: Ulrich Becker/Hans Günter Hockerts/Klaus Tenfelde (Hrsg.), Sozialstaat Deutschland. Geschichte und Gegenwart, Bonn 2010, S. 257-286, hier S. 257.

${ }^{10} \mathrm{Vgl}$. Winfried Schmähl, Sicherung bei Alter, Invalidität und für Hinterbliebene, in: Michael Ruck/Marcel Boldorf (Hrsg.), Geschichte der Sozialpolitik in Deutschland seit 1945, Bd.4: Bundesrepublik Deutschland 1957-1966. Sozialpolitik im Zeichen des erreichten Wohlstandes, Baden-Baden 2008, S. 297-372, hier S. 301.

${ }^{11}$ Das Umlagesystem wurde auch deshalb gewählt, weil der Kapitalstock der Rentenversicherung nach beiden Weltkriegen durch die Inflation völlig entwertet worden war. Vgl. Henning, Staatsfinanzen in historischer Perspektive, S. 58f. und S.61f. Vgl. auch Gerd Hardach, Optionen der Altersvorsorge im 19. und 20. Jahrhundert in Deutschland, in: ZfU 48 (2003), S. 5-28.

${ }^{12}$ Vgl. Philipp Manow, Kapitaldeckung oder Umlage? Zur Geschichte einer anhaltenden Debatte, in: Stefan Fisch/Ulrike Haerendel (Hrsg.), Geschichte und Gegenwart der Rentenversicherung in Deutschland. Beiträge zur Entstehung, Entwicklung und vergleichenden Einordnung der Alterssicherung im Sozialstaat, Berlin 2000, S. 145168. 
Entscheidungsträger das Umlageverfahren. Seit den 1990er Jahren gewinnt das kapitalgedeckte Verfahren wieder mehr Anhänger - kräftig und zweifellos nicht ganz selbstlos unterstützt durch die Finanzindustrie ${ }^{13}$.

Das 1957 festgesetzte Rentenniveau hat sich trotz ständig steigender $\mathrm{Zu}$ schüsse aus dem Bundeshaushalt als nicht finanzierbar erwiesen. Das war Adenauer bereits 1956 von Wilfrid Schreiber, dem wissenschaftlichen Vordenker der Rentenreform, in aller Deutlichkeit gesagt worden. Schreiber hielt nur ein Niveau von 50 Prozent für langfristig finanzierbar ${ }^{14}$. Der Rest sollte durch private Vorsorge, Vermögensbildung und betriebliche Altersvorsorge abgedeckt werden. Bedenken kamen nicht nur aus der Wissenschaft, sondern auch von Finanzminister Schäffer und Wirtschaftsminister Ludwig Erhard (CDU). Beide hielten die Rentenreform für nicht finanzierbar und leisteten erbitterten Widerstand, der den Bundeskanzler zwang, auf seine Richtlinienkompetenz zu pochen ${ }^{15}$.

Das Umlageverfahren und die eingebaute Dynamik funktionierten nur, solange die demographische und ökonomische Entwicklung stimmte. Adenauers oft zitierte Aussage „Kinder bekommen die Leute sowieso ${ }^{\text {“16 }}$ erwies sich als unzutreffend. Der Trend von der Großfamilie zum Single-Haushalt, die Emanzipation der Frau und die Pille als zuverlässiges Mittel zur Empfängnisverhütung haben für stark sinkende Kinderzahlen gesorgt. Doch nichtsdestotrotz wurden 1972 in einer kostspieligen Reform, die ,in einer „bizarren Überbietungskonkurrenz der Parteien zustandekam “'17 , die Rentenleistungen noch einmal deutlich ausgeweitet. „Die Rentenreform von 1972 bezeichnet den Kulminationspunkt einer Entwicklung, in der die Expansion sozialer Leistungen als politisch billiges Nebenprodukt zu haben war. ${ }^{(18}$ Seit der erstmals für 1976 prognostizierten Finanzierungslücke in der Renten-

${ }^{13}$ Vgl. Hockerts, Abschied, S. $276 f$.

${ }^{14}$ Vgl. Wilfrid Schreiber, Existenzsicherheit in der Industriellen Gesellschaft, unveränderter Nachdruck des „Schreiber-Planes“ zur dynamischen Rente aus dem Jahr 1955, Köln 2004, S. 45.

${ }^{15}$ Vgl. Schmähl, Einführung, S. 16, sowie Hockerts, Sozialstaat, S. 12 und S.79. Vgl. allgemein Werner Abelshauser, Erhard oder Bismarck? Die Richtungsentscheidung der deutschen Sozialpolitik am Beispiel der Reform der Sozialversicherung in den Fünfziger Jahren, in: GuG 22 (1996), S.376-392.

${ }^{16}$ Zit. nach Hockerts, Abschied, S. 260.

${ }^{17}$ Hockerts, Sozialstaat, S. 14. Vgl. Hans Günter Hockerts, Vom Nutzen und Nachteil parlamentarischer Parteienkonkurrenz, Die Rentereform 1972 - ein Lehrstück, in: Karl Dietrich Bracher u.a. (Hrsg.), Staat und Parteien. Festschrift für Rudolf Morsey zum 65. Geburtstag, Berlin 1992, S. 903-934.

${ }^{18}$ Hockerts, Sozialstaat, S. 14. 
versicherung wurden die Steigerungsraten und das Leistungsniveau regelmäßig reduziert ${ }^{19}$. In den letzten Jahren der Kanzlerschaft Helmut Kohls (CDU) wurde ein demographischer Faktor eingeführt, den die rot-grüne Bundesregierung nach 1998 abschaffte, dann aber wieder in revidierter Form im Rentenrecht verankerte ${ }^{20}$. Darüber hinaus setzte Finanzminister Hans Eichel mehrere Nullrunden durch. Die dritte große Maßnahme von RotGrün bestand im Aufbau einer privaten, staatlich geförderten Säule der Rentenversicherung, der sogenannten Riester-Rente. Eine Große Koalition hat dann 2006 durchgesetzt, was die Vorgängerregierung nicht mehr geschafft hatte: Die Erhöhung des Renteneintrittsalters auf 67 Jahre. Einen Rückfall in alte Zeiten gab es im Vorwahlkampf des Jahres 2009. Kanzlerin Angela Merkel (CDU) und Arbeitsminister Olaf Scholz (SPD) sorgten nach einer kritischen Mediendebatte dafür, dass die Renten - anders als die Löhne nie sinken dürfen ${ }^{21}$. Bereits zuvor hatte die Große Koalition auf die eigentlich 2005 und 2006 fälligen Rentenabschläge verzichtet.

Insgesamt wurde das Rentenniveau in den letzten drei Jahrzehnten durch große Strukturreformen und weniger sichtbare kleinere Eingriffe wie etwa die Streichung von Ausbildungszeiten deutlich abgesenkt; es dürfte in Zukunft deutlich unter die von Wilfrid Schreiber anvisierte Grenze von 50 Prozent sinken ${ }^{22}$. Ohne dass es ausgesprochen wird, nähert sich die durchschnittliche Rente damit einer Grundrente an, die nicht mehr deutlich über dem Existenzminimum liegt. Die Rentenreformen waren aus finanzpolitischer Sicht durchaus ambitioniert. Ansprüche und damit auch die implizierte Staatsschuld wurden deutlich reduziert ${ }^{23}$. Die Finanzierungslücke

\footnotetext{
${ }^{19}$ Vgl. Winfried Süß, Der keynesianische Traum und seit langes Ende. Sozioökonomischer Wandel und Sozialpolitik in den siebziger Jahren, in: Konrad H. Jarausch (Hrsg.), Das Ende der Zuversicht. Die siebziger Jahre als Geschichte, Göttingen 2008, S. 120-137, hier S. 114f.

${ }^{20} \mathrm{Vgl}$. Hockerts, Abschied, S. 281.

${ }^{21}$ Vgl. Manfred G. Schmidt, Die Sozialpolitik der zweiten Großen Koalition (2005 bis 2009), in: Egle/Zohlnhöfer (Hrsg.), Zweite Große Koalition, S.302-326, hier S.310312 und S. 315.

${ }^{22}$ Vgl. Winfried Schmähl, Ein „Nachhaltigkeitsgesetz“ für die Rentenversicherung. Anspruch und Wirklichkeit, in: Wirtschaftsdienst 84 (2004), S. 210-218, hier S. 216, sowie Moog/Müller/Raffelhüschen, Ehrbare Staaten, S. 10.

${ }^{23}$ Nach Berechnungen des Bundesfinanzministeriums hat sich die Tragfähigkeitslücke, definiert als Summe von impliziter und expliziter Staatsschuld, durch die Einführung des Nachhaltigkeitsfaktors und der Rente mit 67 fast halbiert. Vgl. Zweiter Bericht zur Tragfähigkeit der öffentlichen Finanzen, hrsg. vom Bundesministerium der Finanzen, Berlin 2008, S. 38.
} 
in der Rentenversicherung scheint das erste Mal seit Jahrzehnten beherrschbar zu sein ${ }^{24}$.

Meinhard Miegel und Kurt Biedenkopf warnten bereits vor 30 Jahren vor der „Rentenkrise“ und forderten den Übergang von der lohnbezogenen gesetzlichen Rente zu einer steuerfinanzierten Grundsicherung im Alter, ergänzt durch eine kapitalgedeckte Privatvorsorge $e^{25}$. Im Ergebnis ist Deutschland heute von dieser Forderung gar nicht mehr so weit entfernt, ohne dass es allerdings jemals einen radikalen Systemwechsel gegeben hätte. Das ist wohl auch der Grund dafür, dass die Proteste gegen die Absenkung des Rentenniveaus nicht viel stärker ausgefallen sind.

Die Finanzierungslücke mag zwar kleiner geworden sein, aber dafür wird ein Problem wieder auftauchen, das Adenauers dynamische Rente von 1957 weitgehend gelöst hatte: die Altersarmut. Die „gesetzliche Rentenversicherung hat das Ziel der Lebensstandardsicherung aufgegeben “26, der „Einstieg in den Ausstieg aus der dynamischen Rente mit Lohnersatzfunktion" ist vollzogen ${ }^{27}$. Die Absenkung des Rentenniveaus und die Erosion des Normalarbeitsverhältnisses werden dafür sorgen, dass in Zukunft wohl nicht wenige deutsche Rentner ihr Leben auf Sozialhilfeniveau fristen müssen. Die Sozialhilfe im Alter heißt seit 2003 Grundsicherung; die Ausgaben dafür haben sich seit ihrer Einführung vervielfacht ${ }^{28}$, was bisher vor allem die kommunalen Haushalte belastet hat.

Nicht nur die Grundsicherung ist steuerfinanziert, sondern auch der Zuschuss aus dem Bundeshaushalt zur Rentenversicherung, der eigentlich versicherungsfremde Leistungen ausgleichen soll. Bereits unter Bismarck bezuschusste das Reich die damals neu geschaffene Rentenversicherung. Angesichts der damaligen Alterspyramide, die derjenigen heutiger Schwellenund Entwicklungsländer glich, und der Tatsache, dass die Menschen ihre

\footnotetext{
${ }^{24}$ Vgl. Gisela Färber, Wie ist das Nachhaltigkeitsgesetz zur Rentenversicherung zu beurteilen?, in: Wirtschaftsdienst 84 (2004), S. 207-210, hier S. 210.

${ }^{25}$ Vgl. Meinhard Miegel, Plädoyer für die Weiterentwicklung des Rentensystems, mit einem Vorwort von Kurt H. Biedenkopf, Bonn/Stuttgart 1981. Beide Autoren haben zudem früh vor der Schuldenpolitik gewarnt. Vgl. Meinhard Miegel/Kurt H. Biedenkopf, Die programmierte Krise. Alternativen zur staatlichen Schuldenpolitik, Bonn, Stuttgart 1979. Vgl. auch eine Studie des von Biedenkopf und Miegel gegründeten privaten Forschungsinstituts IWG Bonn: Adrian Ottnad, Wohlstand auf Pump. Ursachen und Folgen wachsender Staatsverschuldung in Deutschland, Frankfurt a. M./ New York 1996.

${ }^{26}$ Hockerts, Abschied, S. 257.

${ }^{27}$ Vgl. Schmähl, Einführung, S. 28.

${ }^{28}$ Vgl. Sozialleistungen der Städte in Not. Zahlen und Fakten zur Entwicklung kommunaler Sozialausgaben, hrsg. vom Deutschen Städtetag, Berlin/Köln 2010, S. $20 \mathrm{ff}$.
} 
Verrentung nicht lange überlebten, waren diese Zuschüsse nicht sonderlich hoch. Das ist heute ganz anders. Allein von 1983 bis 2007, also innerhalb von knapp 25 Jahren, hat sich der Anteil der Rentenausgaben am Bundeshaushalt mehr als verdoppelt. Einige Sonderfaktoren wie die arbeitsmarktpolitisch motivierte Frühverrentung ${ }^{29}$ oder „die relativ hohen Rentenansprüche in den neuen Bundesländern ${ }^{\text {(30 }}$ sind für diese Entwicklung mitverantwortlich. Der Trend der zunehmenden Steuerfinanzierung dürfte auch in Zukunft anhalten, da die Beitragssätze durch die Politik begrenzt worden sind und die Zahlungen aus der Rentenkasse in Folge der demographischen Entwicklung unweigerlich steigen werden.

Neben den Rentenausgaben wird aus dem Bundeshaushalt eine Reihe von weiteren Transferleistungen gezahlt. An erster Stelle ist hier das Arbeitslosengeld (ALG) II zu nennen, bekannter unter dem Namen Hartz IV. Das ALG II ist - wie vormals die Arbeitslosenhilfe - rein steuerfinanziert, also keine Versicherungsleistung. Ein großes Versäumnis der bundesdeutschen Politik bestand darin, diese Tatsache nie richtig kommuniziert und häufig sogar etwas anderes suggeriert zu haben. Ein Teil der Proteste gegen die von der Regierung Schröder im Jahr 2003 durchgesetzte Hartz-Gesetzgebung ${ }^{31}$ rührte genau daher. Viele Menschen haben nicht verstanden, warum der Staat vermeintliche Versicherungsleistungen auf das Sozialhilfeniveau herunterschrauben konnte ${ }^{32}$.

Ein Beitrag zur Haushaltssanierung war die Arbeitsmarktreform der Regierung Schröder nicht. Selbst im Boomjahr 2007, das mit stark sinkenden Arbeitslosenzahlen einherging, gab der Bund für das ALG II knapp 23 Milliarden Euro aus ${ }^{33}$. Hinzu kam noch die Bundesbeteiligung an den Kosten für die Unterkunft der Langzeitarbeitslosen in Höhe von 4,3 Milliarden Euro. Auch die Kommunen, die durch die Einführung von Hartz IV um 2,5 Milliarden Euro entlastet werden sollten ${ }^{34}$, stöhnen über die steigen-

${ }^{29}$ Vgl. Ritter, Vereinigungskrise, S. 171f., sowie Anke Hassel/Christof Schiller, Der Fall Hartz IV. Wie es zur Agenda 2010 kam und wie es weitergeht, Frankfurt a.M. 2010, S. 59-65. Von Hassel und Schiller stammt der Begriff „Stilllegungspolitik“.

${ }^{30}$ Öffentliche Finanzen in der Krise: Ursachen und Handlungserfordernisse, in: Monatsberichte der Deutschen Bundesbank 3/2004, S. 15-37, hier S. 20.

${ }^{31}$ Vgl. Hassel/Schiller, Hartz IV.

${ }^{32}$ Vgl. Manfred G. Schmidt, Sozialpolitik in Deutschland. Historische Entwicklung und internationaler Vergleich, Wiesbaden ${ }^{3} 2005$, S. 120.

${ }^{33}$ Vgl. hierzu und zum Folgenden Bundeshaushalt 2009, Einzelplan 1112, Kapitel 11 (Ist-Ergebnis).

${ }^{34}$ Vgl. Anke Hassel/Christof Schiller, Sozialpolitik im Finanzföderalismus - Hartz IV als Antwort auf die Krise der Kommunalfinanzen, in: PVS 51 (2010), S. 95-117, hier S. 112. 
den Kosten, die letztlich sogar zu Mehrausgaben im Vergleich zur alten Sozialhilfe geführt haben ${ }^{35}$. Insgesamt gab der Bund für die Arbeitsmarkpolitik 200714 Prozent seines Haushalts aus, während der entsprechende Wert für 1983 bei lediglich vier Prozent gelegen hatte.

Eine weitere große Transferleistung ist das Kindergeld. Dieses war von den Nationalsozialisten eingeführt worden, um das Bevölkerungswachstum zu fördern. Die Alliierten hatten es dann abgeschafft, bevor es unter Adenauer 1954 wieder eingeführt wurde ${ }^{36}$. Im Laufe der Jahrzehnte entwickelte sich das Kindergeld zu einer der größten Sozialleistungen des Bundes. Von 1955 bis 1961 gab es ab dem dritten Kind Geld, bis 1974 dann ab dem zweiten und seit 1975 für jedes Kind ${ }^{37}$. Bis 1996 war das Kindergeld für das erste Kind deutlich niedriger als für die anderen Kinder. Seitdem wurde das Kindergeld kräftig erhöht. Insgesamt kostete diese Sozialleistung 2009 mehr als 34 Milliarden Euro ${ }^{38}$. Allein die relative bescheidene Erhöhung um zehn Euro im Jahr 2009 belastet den Haushalt mit jährlich zwei Milliarden Euro. Bundesfinanzminister Peer Steinbrück wollte das Kindergeld nicht erhöhen, sondern stattdessen das Geld zur Finanzierung von zusätzlichen Krippenplätzen umschichten ${ }^{39}$. Damit scheiterte er aber an Familienministerin Ursula von der Leyen (CDU), die ein entschiedenes „sowohl als auch“ durchsetzte, also Kindergelderhöhung und Krippenplätze.

\section{Kriegs- und Verteidigungsausgaben}

Die Verteidigungsausgaben machten im Jahr 2007 zehn Prozent des Bundeshaushalts aus. In historischer Betrachtung darf man getrost sagen: nur zehn Prozent. 1913 dominierten die Militärausgaben den Reichshaushalt mit einem Anteil von 79 Prozent, was allerdings nicht als Beleg für eine besonders kriegerische Mentalität zu sehen ist. Die absoluten Militärausgaben Deutschlands waren nicht höher als diejenigen Großbritanniens, Frankreichs

\footnotetext{
${ }^{35}$ Vgl. Sozialleistungen der Städte, S. $8 \mathrm{ff}$.

${ }^{36}$ Vgl. allgemein Dagmar Nelleßen-Strauch, Der Kampf um das Kindergeld. Grundanschauungen, Konzeptionen und Gesetzgebung 1949-1964, Düsseldorf 2003.

${ }^{37}$ Vgl. hierzu und zum Folgenden Johannes Frerich/Martin Frey, Handbuch der Geschichte der Sozialpolitik in Deutschland, Bd.3: Sozialpolitik in der Bundesrepublik Deutschland bis zur Herstellung der Deutschen Einheit, München/Wien 1993, S. 115-118.

${ }^{38}$ Vgl. Dieter Dohmen/Vera de Hesselle/Andreas Kunzler, Diskrepanz zwischen der demographischen Entwicklung und der Entwicklung der Kindergeldkinder i.S.d. $\$ 32$ Abs. 4 EstG. Gutachten im Auftrag des Bundesfinanzministeriums, Berlin 2009. ${ }^{39}$ Vgl. Der Spiegel vom 26.11.2007, S. 36, und vom 23.06.2008, S. $76 \mathrm{ff}$.
} 
oder Russlands ${ }^{40}$. Aufgrund der föderativen Struktur war der nationale Haushalt Deutschlands nur geringer im Volumen und daher der Anteil der Militärausgaben höher ${ }^{41}$. Anders war die Situation vor dem Zweiten Weltkrieg: Ausmaß und Schnelligkeit der nationalsozialistischen Kriegsvorbereitung bei völliger Missachtung haushälterischer Grundsätze ${ }^{42}$ - dürften in der Weltgeschichte wohl einmalig sein.

1963 beanspruchte der Auf- und Ausbau der Bundeswehr immerhin 35 Prozent des Bundeshaushalts. Der Anteil der Verteidigungsausgaben sank 1983 bis unter zwanzig Prozent, um 2007 schließlich einen historischen Tiefstand zu ereichen. Zweifellos lässt sich hier von einer Friedensdividende nach dem Ende des Kalten Kriegs sprechen. Die Bundesrepublik musste nach 1949 sowohl absolut als auch prozentual nie so viel Geld für Rüstung ausgeben wie die westlichen Siegermächte. Eine Ironie der Geschichte besteht darin, dass die USA die relativ niedrigen deutschen Verteidigungsausgaben heute kritisieren und damit im Grunde auch den Erfolg ihrer eigenen Politik, Deutschland zu einer friedlichen Nation zu erziehen. Angesichts des internationalen Engagements Deutschlands werden die Militärausgaben allerdings wohl wieder steigen.

\section{Zinsbelastungen}

Aufgrund der strukturellen Unterfinanzierung des Reichs und des relativ hohen Zinsniveaus lag die Zins-Ausgaben-Quote 1913 bei neun Prozent. Der niedrige Wert von zwei Prozent im Bundeshaushalt von 1963 war das Ergebnis der weitgehenden Entschuldung in Folge des Staatsbankrotts nach dem Zweiten Weltkrieg sowie einer anfangs äußerst sparsamen Haushaltsführung. Nach der hohen Verschuldung in den 1970er Jahren lag die ZinsAusgaben-Quote im Jahr 1983 bei elf Prozent; bis 2007 kletterte sie um vier Prozentpunkte. Diese relativ niedrige Steigerungsrate ist ganz und gar nicht auf Sparsamkeit zurückzuführen, sondern vor allem auf die historische

${ }^{40}$ Vgl. Henning, Staatsfinanzen in historischer Perspektive, S. 55; Niall Ferguson, Der falsche Krieg. Der Erste Weltkrieg und das 20. Jahrhundert, München ${ }^{2} 2002$, S. $144 \mathrm{f}$.

${ }^{41}$ Bei Addition der Haushalte von Reich und Preußen reduziert sich der Anteil der Militärausgaben für das Jahr 1912 auf 45 Prozent. Vgl. Mark Spoerer, The Evolution of Public Finance in Nineteenth-Century Germany, in José Luis Cardoso/Pedro Lains (Hrsg.), Paying for the Liberal State. The Rise of Public Finance in Nineteenth Century Europe, Cambridge u. a. 2010, S. 103-131, hier S. 113 (Table 4.1).

${ }^{42}$ Vgl. Michiyoshi Oshima, Die Bedeutung des Kabinettsbeschlusses vom 4. April 1933 für die autonome Haushaltsgebarung der Wehrmacht, in: Finanzarchiv 38 (1980), S. 193-235. 
Niedrigzinsphase ${ }^{43}$. Sollten die Zinsen irgendwann wieder steigen, läge darin gewaltiger Sprengstoff für die Staatsfinanzen.

\section{Konjunkturpolitik}

Im weltweiten Krisenjahr 1926 wurde vom deutschen Staat erstmals Konjunkturpolitik betrieben ${ }^{44}$. Im Ergebnis entstand ein strukturelles Defizit, das zusammen mit dem von der Hyperinflation verwüsteten Kapitalmarkt das Deutsche Reich bereits vor der Weltwirtschaftskrise an den Rand der Zahlungsunfähigkeit brachte und die Handlungsspielräume ab 1929 stark einschränkte ${ }^{45}$. Reichskanzler Heinrich Brüning ${ }^{46}$ schaffte auf dem Höhepunkt der Krise den Haushaltsausgleich, indem er die Investitionstätigkeit stark herunterfuhr, die Reparationszahlungen einstellte, die Sozialausgaben kürzte und faktisch auf die Kommunen abwälzte sowie die Löhne und Preise per Notverordnung senkte. Brüning verteidigte zugleich die Goldbindung der Reichsmark und rettete das Bankensystem durch Verstaatlichung. Bis heute wird Brüning vorgeworfen, dass er keine Konjunkturpolitik betrieben, sondern im Gegenteil den wirtschaftlichen Abschwung und die soziale Lage durch seine harte Sparpolitik noch verschärft habe. Daher sei Hitler an die Macht gekommen, der nichts Eiligeres zu tun gehabt habe, als eine keynesianische Konjunkturpolitik umzusetzen und so die Arbeitslosigkeit erfolgreich zu reduzieren ${ }^{47}$.

${ }^{43}$ Zur historischen Entwicklung der Zinssätze vgl. Sidney Homer/Richard Sylla, A History of Interest Rates, Hoboken ${ }^{4} 2005$.

${ }^{44}$ Vgl. Dieter Hertz-Eichenrode, Wirtschaftskrise und Arbeitsbeschaffung. Konjunkturpolitik 1925/26 und die Grundlagen der Krisenpolitik Brünings, Frankfurt a.M./New York, 1982, sowie Fritz Blaich, Die Wirtschaftskrise 1925/26 und die Reichsregierung. Von der Erwerbslosenfürsorge zur Konjunkturpolitik, Kallmünz 1977.

${ }^{45}$ Vgl. Heike Knortz, Wirtschaftsgeschichte der Weimarer Republik, Göttingen 2010, S. 160-165 und S. 206.

${ }^{46}$ Vgl. Herbert Hömig, Brüning. Kanzler in der Krise der Republik. Eine Weimarer Biographie, Paderborn u.a. 2000, sowie William L. Patch, Heinrich Brüning and the Dissolution of the Weimar Republic, Cambridge 1998.

${ }^{47}$ Selbst Keynes scheint den Charakter der NS-Finanz- und Wirtschaftspolitik verkannt zu haben: „Nevertheless the theory of output as a whole, which is what the following book purports to provide, is much more easily adapted to the conditions of a totalitarian state." John Maynard Keynes, The General Theory of Employment, Interest and Money, Cambridge 1936 (ND 1993), S.XXVI (Preface to the German Edition). Das Deficit spending der Nationalsozialisten hatte jedoch nicht einen sich selbst tragenden Wirtschaftsaufschwung als Ziel, sondern die Finanzierung eines Angriffskriegs, war also keineswegs keynesianisch. Vgl. Richard J. Overy, The Nazi Economic Recovery 1932-1938, London 1982. 
Um die Bewertung der Finanzpolitik Brünings wird in der Wissenschaft seit Jahrzehnten erbittert gestritten ${ }^{48}$. Ein Konsens konnte bisher nicht hergestellt werden. Die Deflationspolitik Brünings mag Ausdruck einer rationalen Strategie ${ }^{49}$ gewesen sein und möglicherweise die Grundlagen für den folgenden Wirtschaftsaufschwung ${ }^{50}$ gelegt haben. Im Ergebnis vertiefte sie jedoch die deutsche Staats- und Wirtschaftskrise auf fatale Weise. Es ist nicht sicher, ob die Wirtschafts- und Finanzkrise, die 2008 eingesetzt hat, der Kontroverse neue Impulse gibt. Zum einen wird als Lehre aus der Weltwirtschaftskrise ein aktives Gegensteuern abgeleitet. So flutete Ben Bernanke, der amerikanische Notenbankenpräsident und einer der führenden Wirtschaftshistoriker $^{51}$, die Geldmärkte mit Liquidität ${ }^{52}$ und empfahl seiner Regierung, gigantische Konjunkturprogramme aufzulegen. Zum anderen kann seit den jüngsten Schwierigkeiten von Griechenland deutlicher verstanden werden, dass Brüning wirklich in einer „Zwangslage“ steckte, weil die öffentliche Hand keine Kredite mehr erhielt. Insofern war der Haushaltsausgleich, also der Verzicht auf Neuverschuldung, die einzig verbleibende Option.

Konjunkturpolitik hat eine Renaissance gefeier ${ }^{53}$. Das ist erstaunlich, weil Konjunkturprogramme bis vor kurzem als völlig wirkungslos galten. Im internationalen Vergleich wurde auf Japan verwiesen, das in den 1990er

${ }^{48}$ Hauptopponenten dieser Kontroverse sind Knut Borchardt und Carl-Ludwig Holtfrerich. Vgl. den Überblick bei Theodore Balderston, Economics and Politics in the Weimar Republic, Cambridge 2002, S.77-99, sowie bei Albrecht Ritschl, Knut Borchardts Interpretation der Weimarer Wirtschaft. Zur Geschichte und Wirkung einer wirtschaftsgeschichtlichen Kontroverse, in: Jürgen Elvert/Susanne Krauß (Hrsg.), Historische Debatten und Kontroversen im 19. und 20. Jahrhundert, Stuttgart 2001, S. 234-244.

${ }^{49}$ Vgl. z.B. Albrecht Ritschl, Deutschlands Krise und Konjunktur 1924-1934. Binnenkonjunktur, Auslandsverschuldung und Reparationsproblem zwischen Dawes-Plan und Transfersperre, Berlin 2002.

${ }^{50}$ Vgl. Christoph Buchheim, Die Wirtschaftsentwicklung im Dritten Reich - mehr Desaster als Wunder. Eine Erwiderung auf Werner Abelshauser, in: VfZ 49 (2001), S.653-664; Werner Abelshauser, Kriegswirtschaft und Wirtschaftswunder, in: VfZ 47 (1999), S. 1-61. Vgl. auch Mark Spoerer, Demontage eines Mythos? Zu der Kontroverse über das nationalsozialistische „Wirtschaftswunder“, in: GuG 31 (2005), S.415438.

${ }^{51}$ Vgl. Ben S. Bernanke, Essays on The Great Depression, Princeton 2000.

${ }^{52}$ „Bernanke had well learned the lesson from the banking panics of the 1930s of conducting expansionary monetary policy to demands for liquidity." Michael Bordo/ John Landon-Lane, The banking panics in the United States in the 1930s: some lessons for today, in: Oxford Review of Economic Policy 26 (2010), S. 486-509, hier S. 486.

${ }^{53}$ Vgl. Thomas Straubhaar/Michael Wohlgemuth/Joachim Zweyner, Rückkehr des Keynesianismus, in: APuZ 20/2009, S. 19-26. 
Jahren ein Konjunkturprogramm nach dem anderen auflegte, um aus einer deflationären Wirtschaftskrise herauszukommen ${ }^{54}$. Wirtschaftspolitisch war dieser Kurs nur mäßig erfolgreich, finanzpolitisch sogar verheerend. Die japanische Schuldenquote explodierte von 1990 bis 2000 von 68 auf 142 Prozent $^{55}$. Wohl noch nie hat sich ein Staat, der keinen Krieg führte, in so kurzer Zeit so hoch verschuldet. Das Ergebnis deckte sich mit den deutschen Erfahrungen in den 1970er Jahren, als die Konjunkturpolitik weitgehend wirkungslos verpuffte, aber eine hohe Staatsverschuldung hinterlie $\beta^{56}$. Peer Steinbrück lehnte daher Konjunkturprogramme vehement ab, vermochte sich mit dieser Haltung allerdings nicht durchzusetzen ${ }^{57}$.

Helmut Schmidt (SPD) hatte in seiner Kanzlerschaft zwischen 1974 und 1982 versucht, die Konjunktur mit insgesamt weit über 60 Milliarden DM anzukurbeln $^{58}$. Mit dieser Strategie vermochte er die "Stagflation“ - eine Mischung aus wirtschaftlicher Stagnation und hoher Inflation - jedoch nicht zu überwinden. Es blieb vor allem ein Ergebnis: Die Staatsschulden, die bis dahin relativ niedrig gewesen waren, wuchsen ab 1973/74 beinahe exponentiell an. Wohl nicht zuletzt aufgrund des Erfolgs der ersten Großen Koalition im Krisenjahr 1966/67 hielt Helmut Schmidt so lange an dieser teuren Politik fest ${ }^{59}$. Der ersten bundesdeutschen Rezession wurde unter Füh-

${ }^{54}$ Vgl. Toshihiro Ihori/Torun Nakazato/Masumi Kawade, Japan's Fiscal Policies in the 90s, in: Gary R. Saxonhouse/Robert M. Stern (Hrsg.), Japan's Lost Decade. Origins, Consequences and Prospects for Recovery, Malden u. a. 2004, S. 59-72.

${ }^{55}$ Vgl. Monatsbericht des Bundesministeriums der Finanzen vom Januar 2011, S. 87.

${ }^{56}$ So das einmütige Urteil der Wissenschaft. Zusammenfassung bei Claus-Martin Gaul, Konjunkturprogramme in der Geschichte der Bundesrepublik Deutschland: Einordnung und Bewertung der Globalsteuerung von 1967 bis 1982, Berlin 2009 (www. bundestag.de/dokumente/analysen/2009/konjunkturprogramme.pdf). Vgl. z.B. Tim Schanetzky. Die große Ernüchterung, Wirtschaftspolitik, Expertise und Gesellschaft in der Bundesrepublik 1966 bis 1982, Berlin 2007; Monika Hanswillemenke/Bernd Rahmann, Zwischen Reformen und Verantwortung für Vollbeschäftigung. Die Finanzund Haushaltspolitik der sozial-liberalen Koalition von 1969 bis 1982, Frankfurt a.M. 1997; Alexandra Ehrlicher, Die Finanzpolitik 1967-1976 im Spannungsfeld zwischen konjunkturpolitischen Erfordernissen und Haushaltskonsolidierung, Berlin 1991; Harald Scherf, Enttäuschte Hoffnungen - vergebene Chancen. Die Wirtschaftspolitik der Sozial-Liberalen Koalition 1969-1982, Göttingen 1986.

${ }^{57}$ Vgl. Henrik Enderlein, Finanzkrise und große Koalition: Eine Bewertung des Krisenmanagements der Bundesregierung, in: Egle/Zohlnhöfer (Hrsg.), Zweite Große Koalition, S. 234-253, hier S. 239-244.

${ }^{58}$ Vgl. Gaul, Konjunkturprogramme, S. 14.

${ }^{59}$ Vgl. Hartmut Soell, Helmut Schmidt: Zwischen reaktivem und konzeptionellem Handeln, in: Jarausch (Hrsg.), Ende der Zuversicht, S. 279-295. 
rung von Wirtschaftsminister Karl Schiller (SPD) und Finanzminister Franz Josef Strauß (CSU), damals Plisch und Plum genannt, ein Konjunkturprogramm in Höhe von knapp acht Milliarden DM entgegengesetzt. Die Krise war schnell überwunden, und die Verschuldung wurde danach mustergültig abgebaut. Franz Josef Strauß schrieb Ende der 1960er Jahre ein Buch, das sich wie eine Anleitung für erfolgreiche Keynesianer liest ${ }^{60}$. Schiller und Strauß waren damals davon überzeugt, die Konjunktur steuern zu können. Wörtlich formulierte Schiller: „Konjunktur ist nicht unser Schicksal, Konjunktur ist unser Wille. ${ }^{{ }^{6} 11}$ Der wortgewaltige Wirtschaftsminister, ordentlicher Professor der Volkswirtschaftslehre, war davon überzeugt, mit seiner im Stabilitätsgesetz verankerten Globalsteuerung ein fine-tuning der Wirtschaft zu erreichen ${ }^{62}$. Ohne dass er dies wollte, weckte sein keynesianischer Steuerungsoptimismus ein Vertrauen in den Staat, das letztlich zu einer deutlichen „Erweiterung des Staatskorridors“ führte ${ }^{63}$. Insofern war Schiller, der für kurze Zeit auch Finanzminister war, eine tragische Gestalt der deutschen Finanzgeschichte ${ }^{64}$.

Der Erfolg von 1966/67 wiederholte sich in den 1970er Jahren nicht ${ }^{65}$. Konjunkturpolitik erwies sich als die falsche Antwort auf die Ölpreiskrisen von 1973/74 und 1979 sowie auf die strukturelle Verschiebung der Weltwirtschaft in Richtung Fernost. Damals wäre eine angebotsorientierte Wirtschaftspolitik mit dem Ziel einer Steigerung der internationalen Wettbewerbsfähigkeit die adäquatere Strategie gewesen. In einem quälend langen Prozess vollzog sich die Loslösung vom Keynesianismus bereits 1979 unter Finanzminister Hans Matthöfer (SPD) ${ }^{66}$, der „die Hinterlassenschaften einer falsch verstandenen ,Keynesianischen Revolution“ stillschweigend ${ }^{\text {(67 }}$ beerdigte.

\footnotetext{
${ }^{60}$ Vgl. Franz Josef Strauß, Finanzpolitik. Theorie und Wirklichkeit, Berlin 1969.

${ }^{61}$ Zit. nach Gaul, Konjunkturprogramme, S. 16.

${ }^{62}$ Vgl. Nützenadel, Stunde der Ökonomen, S. 307-343.

${ }^{63}$ Hans-Peter Ullmann, Im Strudel der „Maßlosigkeit“? Die „Erweiterung des Staatskorridors" in der Bundesrepublik der sechziger bis achtziger Jahre, in: ders., Staat und Schulden. Öffentlichen Finanzen in Deutschland seit dem 18. Jahrhundert, hrsg. von Hartmut Berghoff und Till van Rahden, Göttingen 2009, S. 149-162.

${ }^{64}$ Karl Schiller war 1967-1972 Bundeswirtschaftsminister und 1971/72 zusätzlich Bundesfinanzminister; vgl. Torben Lütjen, Karl Schiller (1911-1994). „Superminister“ Willy Brandts, Bonn 2007.

${ }^{65}$ Vgl. Nützenadel, Stunde der Ökonomen, S. 344-352.

${ }^{66}$ Vgl. Werner Abelshauser, Nach dem Wirtschaftswunder. Der Gewerkschafter, Politiker und Unternehmer Hans Matthöfer, Bonn 2009.

${ }^{67}$ Werner Abelshauser, Aus Wirtschaftskrisen lernen - aber wie? Krisenszenarien im Vergleich, in: VfZ 57 (2009), S. 467-483, hier S. 480.
} 
Mit der „Wende ${ }^{\text {c68 }}$ von 1982 wurde der Monetarismus von Milton Friedman zum Leitstern der Wirtschaftspolitik. Oskar Lafontaine (SPD) versuchte 1998 eine Kehrtwende zum Keynesianismus, scheiterte damit aber an Bundeskanzler Schröder.

\section{Personalausgaben}

Im Jahr 2007 betrug der Anteil der sonstigen Ausgaben am Bundeshaushalt 23 Prozent. Dabei handelt es sich um ein Sammelsurium von Positionen: Von der Entwicklungshilfe bis zur Förderung der Forschung und diversen Wirtschaftssubventionen. Auch die Personal- und Sachausgaben für die Bundesverwaltung fallen im Wesentlichen hierunter. Insgesamt machen die Personalausgaben weniger als zehn Prozent des Bundeshaushalts aus ${ }^{69}$. Zum Vergleich: Bei den Kommunen liegt der Anteil bei ungefähr 25 Prozent $^{70}$, während die westdeutschen Länder sogar eine (bereinigte) Personalausgabenquote von 37 Prozent $^{71}$ aufweisen. Die Bundesministerien verfügen zwar über viele hoch dotierte Stellen, sind im internationalen Vergleich aber relativ klein. So hat das Bundesfinanzministerium in der Berliner Wilhelmstraße zwar ein imposantes Gebäude bezogen, welches seinerzeit großspurig von Hermann Göring geplant worden war $^{72}$, doch verglichen mit seinem französischen Pendant in Paris-Bercy nimmt es sich geradezu bescheiden aus. Deutschland ist ein föderaler Staat, in dem ein Großteil des operativen Verwaltungshandelns von Ländern und Kommunen geleistet wird. Das spiegelt sich in den Personalkörpern wider.

Wie sehr der Staatsapparat in den 1960er und vor allem in den 1970er Jahren ausgebaut wurde, zeigt der Anstieg der Beschäftigtenzahlen bei Bund, Ländern und Kommunen von 1,9 Millionen 1960 auf 3,3 Millionen 1980 $0^{73}$. Die öffentlichen Aufgaben stiegen damals sowohl in ihrer Quantität als auch

\footnotetext{
${ }^{68}$ Vgl. Klaus Zimmermann, Zur Realität von Kurswechseln, Wenden und ähnlichen Manövern aus budgetpolitischer Sicht, in: Der Gemeindehaushalt 9 (1984), S. 208-213.

${ }^{69}$ Der Anteil der Personalausgaben an den gesamten Ausgaben des Bundeshaushalts ist in den letzten Jahrzehnten stark gesunken. Von 16 Prozent 1975, über 15 Prozent 1980 und 11 Prozent 1990 und 2000 auf neun Prozent 2010. Vgl. Monatsbericht des Bundesministeriums der Finanzen vom Januar 2011, S. 74f .

${ }^{70}$ Vgl. Gemeindefinanzbericht 2010, in: Der Städtetag 5/2010, S. 5 (eigene Berechnung).

${ }^{71}$ Vgl. Monatsbericht des Bundesministeriums der Finanzen vom Januar 2011, S. 92 (eigene Berechnung).

${ }^{72}$ Vgl. Laurenz Demps/Eberhard Schultz/Klaus Wettig, Bundesfinanzministerium. Ein belasteter Ort?, Berlin 2002.

${ }^{73}$ Vgl. Norbert Andel, Finanzwissenschaft, Tübingen ${ }^{4}$ 1998, S. 207.
} 
in der Qualität. Beispiele sind die Gründung von Hochschulen und der Ausbau des Schulsystems, die einen immensen Mehrbedarf an Personal mit sich brachten.

Unter dem Druck der defizitären Haushalte war es mit der großzügigen Einstellungspraxis spätestens mit Beginn der 1980er Jahre vorbei. Erst die Wiedervereinigung führte durch die aufgeblähten Verwaltungsapparate der DDR zu einem sprunghaften Anstieg der öffentlich Beschäftigten. Besonders drastisch war die Situation in Berlin. Das über Jahrzehnte hoch subventionierte West-Berlin hatte einen unangemessen großen Verwaltungsapparat aufgebaut und vereinigte sich 1990 mit der noch größeren Bürokratie OstBerlins. Erst und ausgerechnet ein rot-roter Senat senkte die Beschäftigtenzahlen drastisch. Seit den 1990er Jahren stehen die Vorzeichen im öffentlichen Dienst generell auf Abbau, und zwar nicht nur in Ostdeutschland. Um Kosten zu sparen, haben sämtliche Gebietskörperschaften in den letzten 20 Jahren massiv Personal abgebaut. Diese Leistung ist bemerkenswert, da die Anzahl der Aufgaben und die Anforderungen an die Qualität der öffentlichen Dienstleistungen nicht kleiner geworden sind. 
